\title{
CHALLENGES OF TEACHING SOUND STUDIES THAT INCLUDE PRESERVATION ISSUES IN MALAYSIA
}

Ahmad Faudzi Musib, Universiti Putra Malaysia, Malaysia

Gisa Jähnichen, Shanghai Conservatory of Music, China

\section{Introduction}

At the Music Department of Universiti Putra Malaysia, that since 2 years offers universitywide the opportunity for audiovisual archiving in ARCPA (Audiovisual Research Collections of Performing Arts), the implementation of a course named 'Sound Studies" is planned.

If we say 'planned', it means that in the light of shaping a better profile of each of the few tertiary music education units within Malaysia, each of these units should find some special focus that enriches the academic landscape and attracts industrial attentiveness. If done in an effective way, students from all over the region can be educated on high level and serve then as multipliers in their local homes or home countries as audiovisual archivists.

Sound Studies as a university subject is not new though relatively new to Malaysia.

Some dedicated composers cum sound engineers tried to get into the field of sound studies in order to provide creative tools to be used for musicians or for stage performances such as Hasnizam Abdul Wahid (UNIMAS, University Malaysia Sarawak) or Mohammad Azam Sulong (UPSI, Universiti Pendidikan Sultan Idris) from various educational institutions in Malaysia. The idea, however, that sound studies may cover human ecology of sound and within this area a science on preservation approaches to sound and audiovisual material is new.

\section{Main problem}

One of the big problems is that practical aspects of sound are seemingly strictly separated from reflective academia.

A recent paper by Chan claims to be the first on this topic. He seems to separate groups of people affected such as students from non-students: "The objective of this paper is to determine if the present state of noise exposure in the Malaysian living environment can be both a research and practical problem from the perspective of music education, by looking into the quality of the noise and its implications on the growth of a person." (Chan, 2015: 32).

The paper comes later up with some insights on conditions in "indoor rooms" where ceiling fans and air conditions are "heavily" used and compared with the given standards on occupational health that may impose (I) a physical threat to the student's growth and, obviously to his surprise, (2) a threat to the life quality of the student (ibid: 34 ) using a former stated theoretical framework from another scholar who had some quite different goals.

The method how such studies are produced in the academic world makes wild measurement collections of "soundscapes", school corridors, and even "indoor rooms" a numerical necessity in order to produce written texts on facts that are practically known to everyone. In its concluding remarks, Chan recommends teachers to send students with hearing difficulties to the medical doctor, to draw their attention to "soundscapes", which are of course out there somewhere, and to use identified soundscape elements for creative performances. 


\section{Other problems}

The most interesting of the article (Chan 20I5) is, however, that nearly all problems faced in order to get to the point of promoting a truly advanced course on sound studies are concentrated and factually demonstrated within the text:

- The priority of technical equipment that completely replaces any social and historical approach to what sound means in an actual place and among actual people;

- The priority of formal settings given through standard rules for measurements as well as for writings;

- The eclecticism of perspectives and the quasi-scientific choice of data that fit the technical equipment;

- The importance of being Malaysian while researching sound in Malaysia;

- The hiding behind educational matters in the tone of being worried about something that does not affect anyone except the young generation;

- The obvious tendency not to question the cause of a danger but the delivered reports on it, which makes academicians ultra-cautious - Prufockian - in naming social causalities;

- The deeply ingrained unawareness of sound as a social, historical and individual belonging that permeates any type of environment.

In other words: the students get deaf because they weren't aware of the danger. So, it's their fault. And teachers have to guide the students in becoming aware of it, if possible through playing around with 'soundscapes'. That would then make a good teacher as we learn from Chan's article.

\section{Change of perspectives}

Things are different, if they are looked at from a global or at least regional perspective. ARCPA', the small research oriented university audiovisual archive established at the UPM ${ }^{2}$ music department was an issue that had to be defended (see earlier reports: Musib, Jähnichen \& Meddegoda 20I4; Jähnichen 20I5).

If the establishment of ARCPA was already a great effort not only from the technical aspect but from the aspect of changing attitudes and mind sets within the academic environment of the largest public Malaysian University, one could wonder what the new course will mean to the involved parties.

While audio engineering and 'digital creativity' is a widely accepted business, it seems to be tremendously important to also attract interest into sound studies that include preservation of sound recordings, since a vast amount of recordings available in the region are shortly before their expiry date.

The cultural situation in Malaysia nurtured over many decades a quite careless dealing with those resources that are stored under difficult conditions. Latest by now, it is time to improve the situation through educating man power and - at the same time - through teaching future users of documents preserved in AV archives. 
Finally, taught preservation efforts in accordance with an increasing awareness of sound as an environmental condition is examined in this paper based on philosophical as well as actual observations made during experiments in Padawan, Sarawak, between 2012 and 20I5. The following scheme summarizes the many factors influencing the establishment of 'Sound Studies' as a tertiary educational subject in Malaysia ${ }^{3}$ :

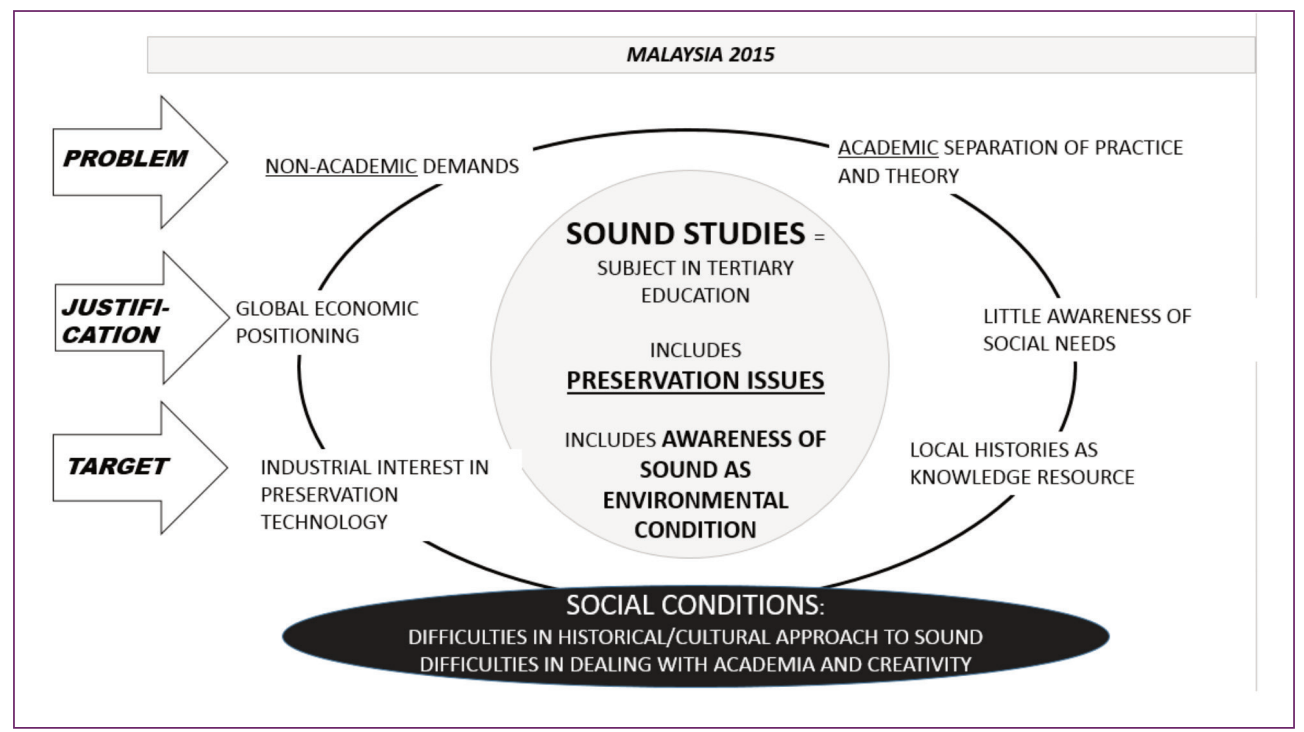

Figure I: Scheme of influencing factors on the introduction of Sound Studies as subject in tertiary education (Malaysia).

\section{Attracting attention}

A first attempt is to draw the attention of non-academicians to the possibilities that an audiovisual archive can provide in order to serve the community. This point can't be over-emphasized as this is a crucial moment in justifying audiovisual archiving.

Recently, Ahmad Faudzi Musib developed an effective work flow for contextual sound preservation of selected string instruments in rural and urban areas of Borneo. To be more precise, in Anah Rais, a small longhouse village of Padawan, a subregion of Greater Kuching, Sarawak. $\mathrm{He}$ applied new aspects of high resolution recordings and technical highlights set into a spatial framework, which are worth to be shared. In connection with his study that is explained in the second part, I am going to further develop this approach and expand it regarding variously constituted definitions of 'musical life' within a selected space and time.

Musib produced sound analytical findings that help to understand how the concept of a "sounding home" works among people with different cultural conditions. Core questions are whether contextual sound is indicative for a spatial sound memory and if so, which role plays the "musical life' of a place in a time through amplifying recognition patterns and creative impulses.

The principle is easy to understand and to explain: Humans are used to listen to the entire sound offered. However, attention is mostly paid to those sounds that are considered to be of relevance in the flow of conscious living. They contribute to orientation, decision making and patterns of response within a communication network. 
Musical life is part of it. Musical life as embedded in sound events can be seen as contextual sound apart from primary attention.

The problem discussed here is the factual division of foreground or focus and background or contextual environment. Further, the discussion is about how sound may contribute to patterns of human movements, to individual decisions and to spatial memory. It is recommended to eventually use perspectivism as discussed in Heonik Kwon's paper on "Perspectivism in Social Anthropology" (20I2). It can help to compensate for some weaknesses that place the rather technical 'quality of recorded sound' into the centre of any preservation efforts. ${ }^{4}$

The experiments done in a village of the Bidayuh situated in Padawan on Borneo show that contextual sound preservation is an important tool in tracing cultural memory and in placing oneself into a local history which includes present and possible future.

Preserving a sound context in order to cater additional information needs for the future is not a new issue. Most of academic writings do so since the very beginning of musicology. But they do it without sound. Places, times and agents are described in a rather static way as words can't indicate the same information compared to sound which is in its nature a dynamic and a moving feature strictly bound to an actual time and a real place.

Listening to recorded sound triggers the re-construction of time and place. If there is no experience for that, people do individually imagine time and place. If they do not imagine, the technical device such as a CD player or the CD rack from where the sound carrier was taken one day becomes the time and place reconstructed.

Here, a model is developed that includes sound environmental perspectives. The main question is 'How do these people [horizontal categories] here those people [vertical categories]'. The answers were collected through observation and informal conversation with the villagers. Most interesting are the individually different sound memory patterns that are introduced following this first scheme.

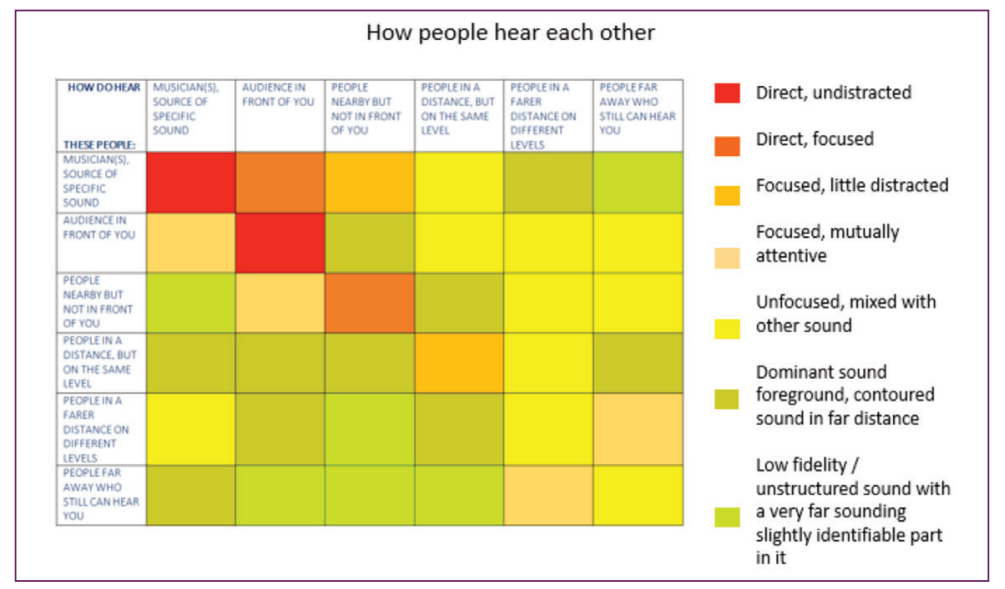

Figure 2: How people hear each other.

The range of focus, distracting sound, contoured sound, dominant sound and identifiable parts in it is large and individually different depending on the personal profile of a community member or a community group. In some cases, memory patterns can follow the focus on the main source and transitional perceptions from tolerance to disturbance. 


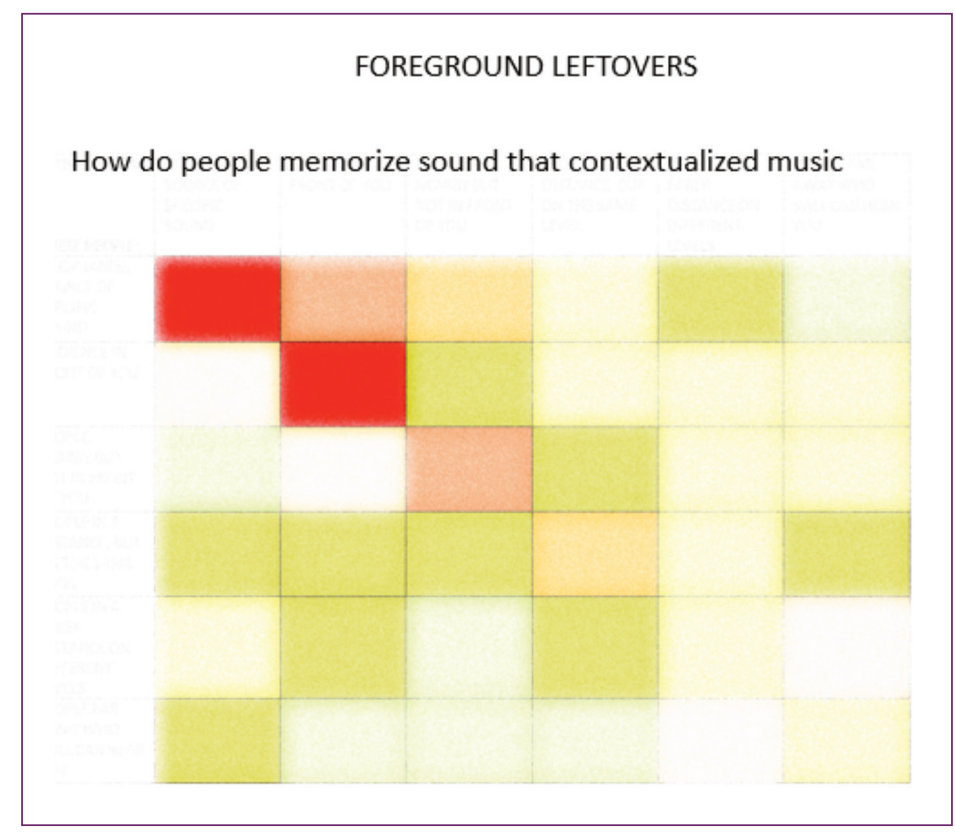

Figure 3: How do people memorize sound that contextualized music (I).

Other individual memory patterns may completely delete stronger transitional perceptions.

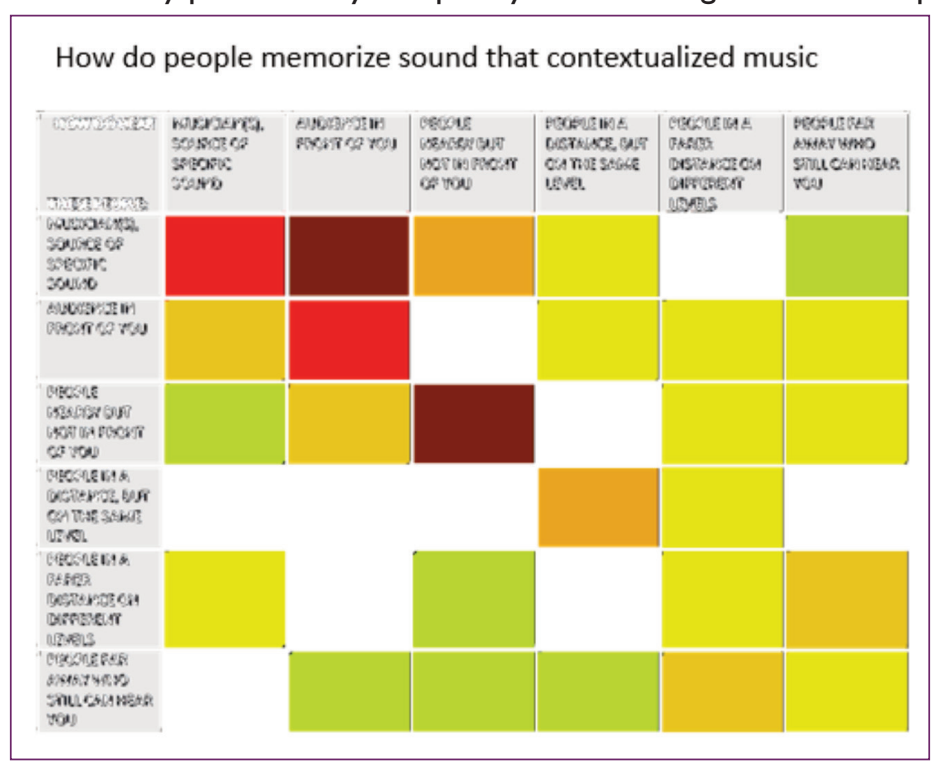

Figure 4: How do people memorize sound that contextualized music (2). 
Again other memory patterns show indistinct and overlapping structures that include a number of unequal perceptions as a whole construction.

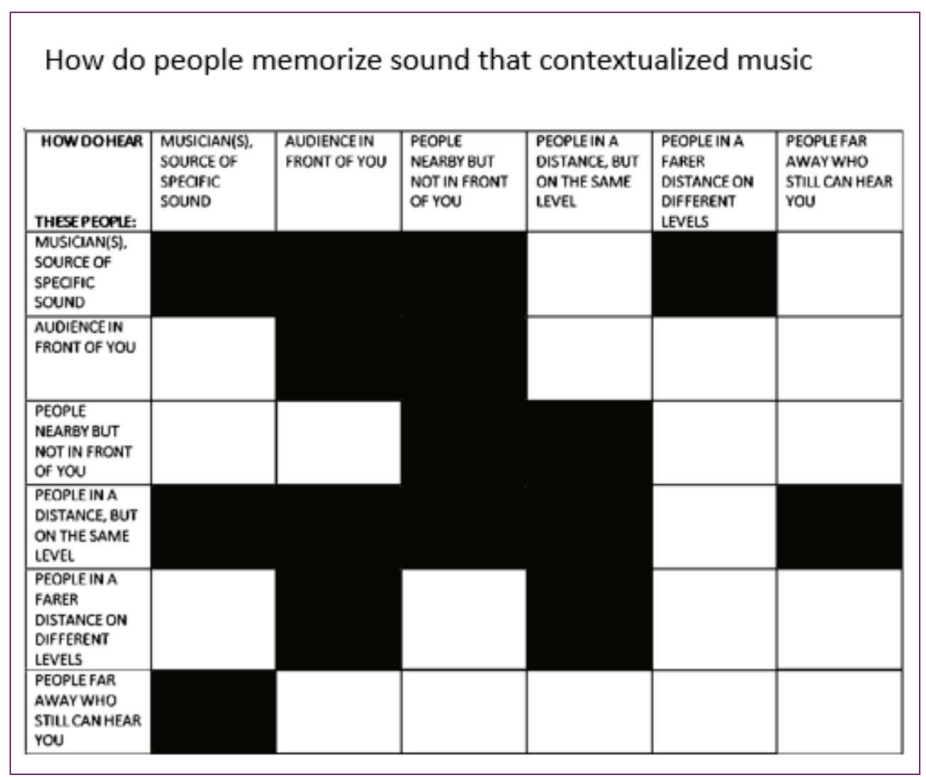

Figure 5: How do people memorize sound that contextualized music (3).

Or the memory draws on so called 'cleaned' recordings that are found on world music cassettes and CDs. By repeatedly listening to these recordings, the entire contextual sound is hidden from being traced as a stimulus.

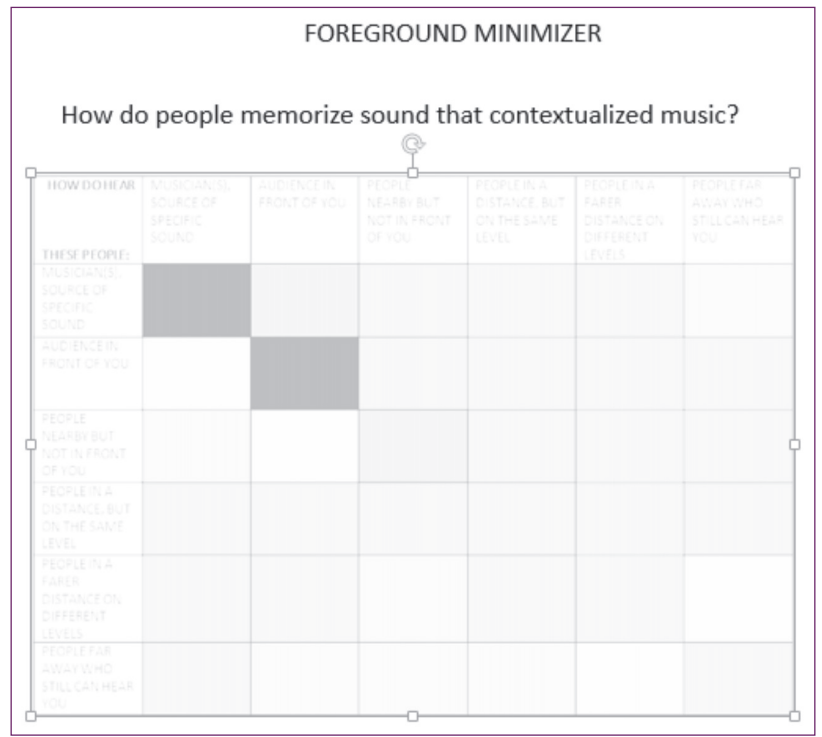

Figure 6: How do people memorize sound that contextualized music (4). 
Most people who have a live experience with the sound source will add a blurry context to the cleaned sound.

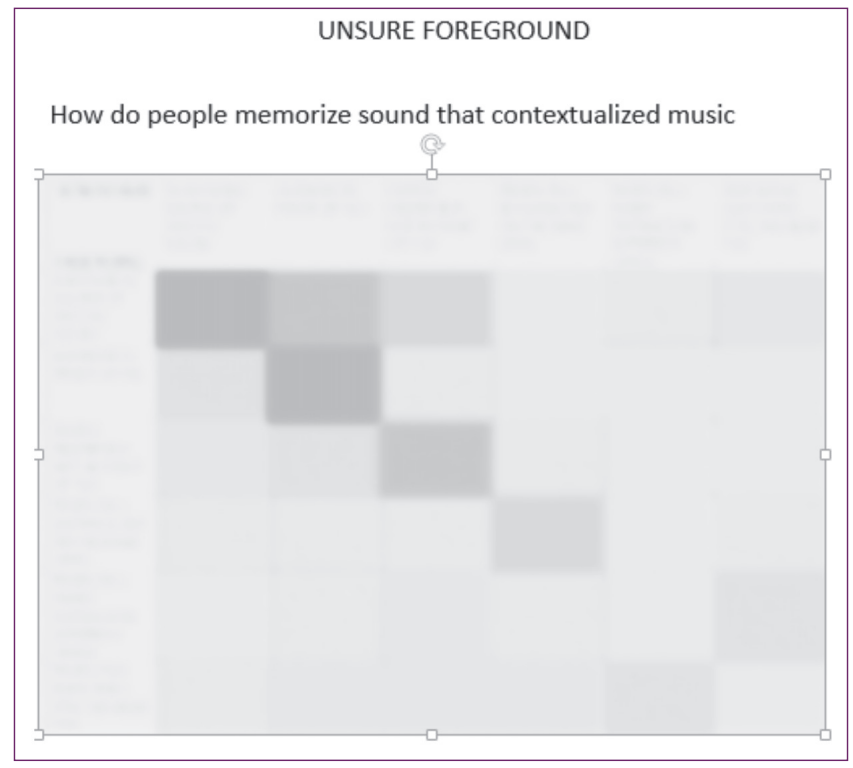

Figure 7: How do people memorize sound that contextualized music (5).

Howsoever those memories work with sound sources, the initial scheme that represents already an abstract summary is increasingly vulnerable through modifying sound re-production. Also, the scheme applies only on people who were or are familiar with the main sound source and had the opportunity to observe different constellations at different places and in different times. And yet, different individual perspectives will emphasize different perspectives:

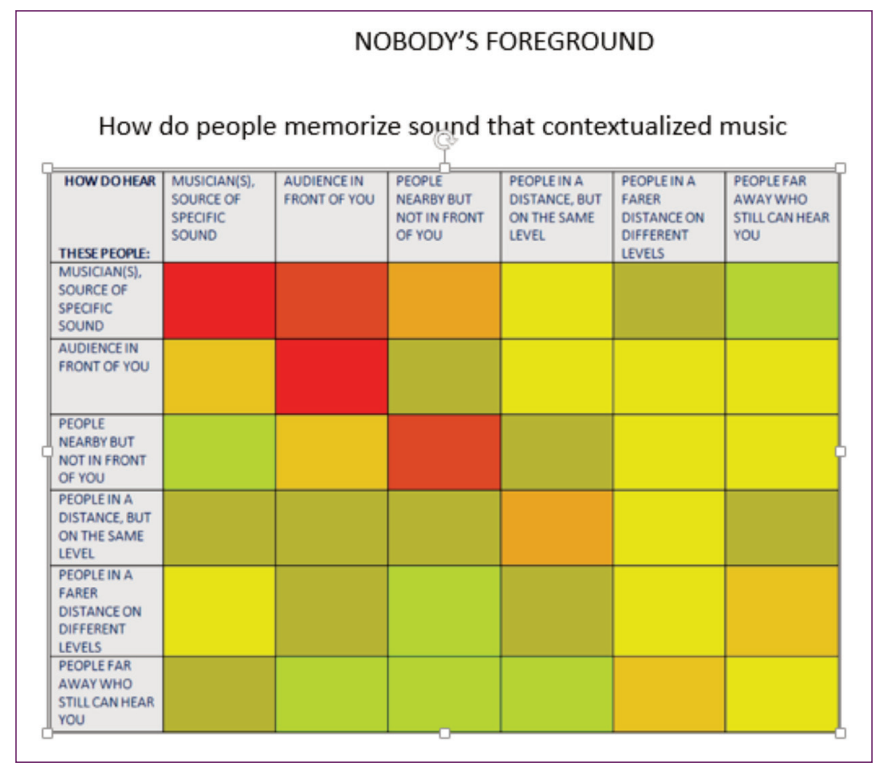

Figure 8: How do people memorize sound that contextualized music (6). 
Finally, each actively involved participant has another hearing profile and differentiates sound in various ways. These profiles have definitely an impact on the sound memory within a community or a group of people, not only how they hear, but how they are heard.

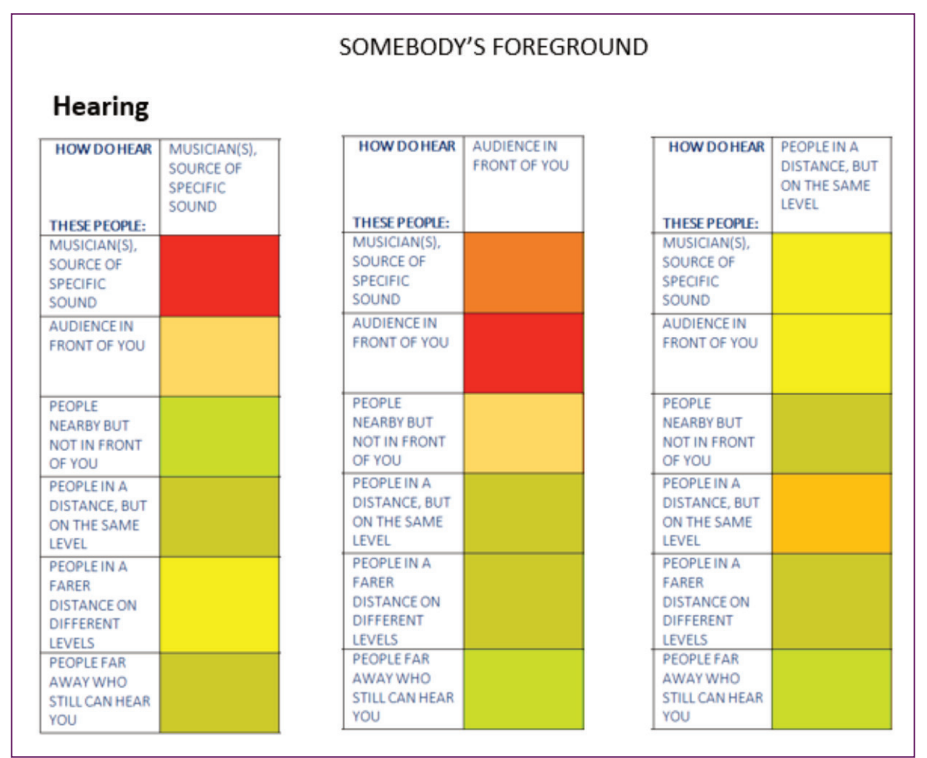

Figure 9: Hearing - somebody's foreground.

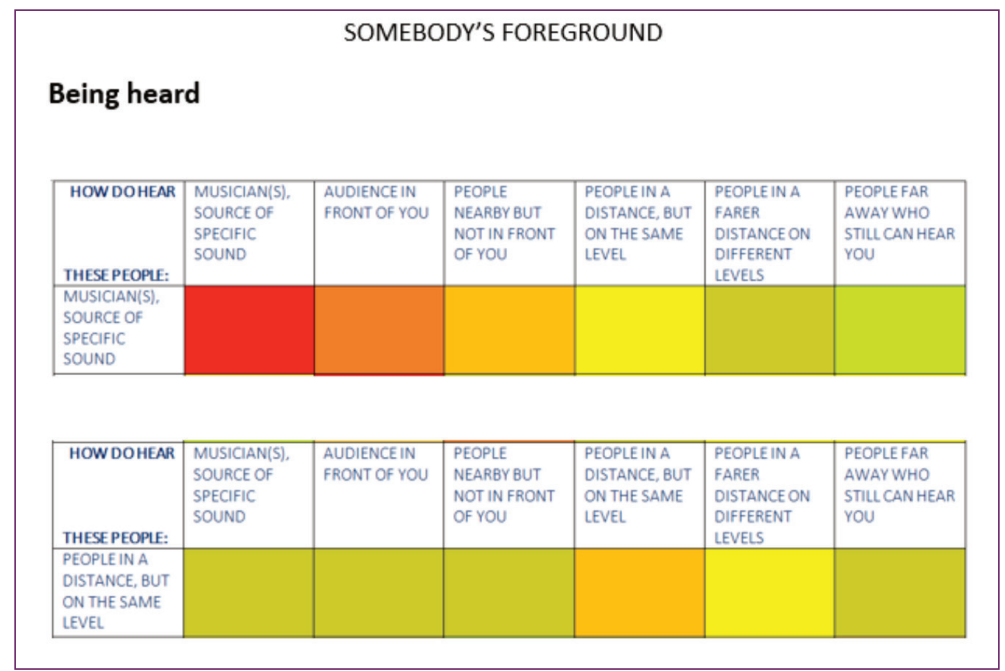

Figure 10: Being heard - somebody's foreground.

Sound memories differ according to the frequency of sound awareness in relation to a spatial order as here between a farmer, a musician and a homeworker. 
Putting the outcomes of the study into its historical context and summarizing dynamics of changes in the meaning and practice of specific sound as the sound of the pratuokng ${ }^{5}$, obviously many 'sounds' if not all are inter-connective throughout the times. They can be emblematic, surrogating or pragmatic, they can be just habitual, they can be newly added sounds. Whatever they are and under which aspect or using which theory they exist, they are connected to other sounds, even though nobody knows, even without setting an ecological framework.

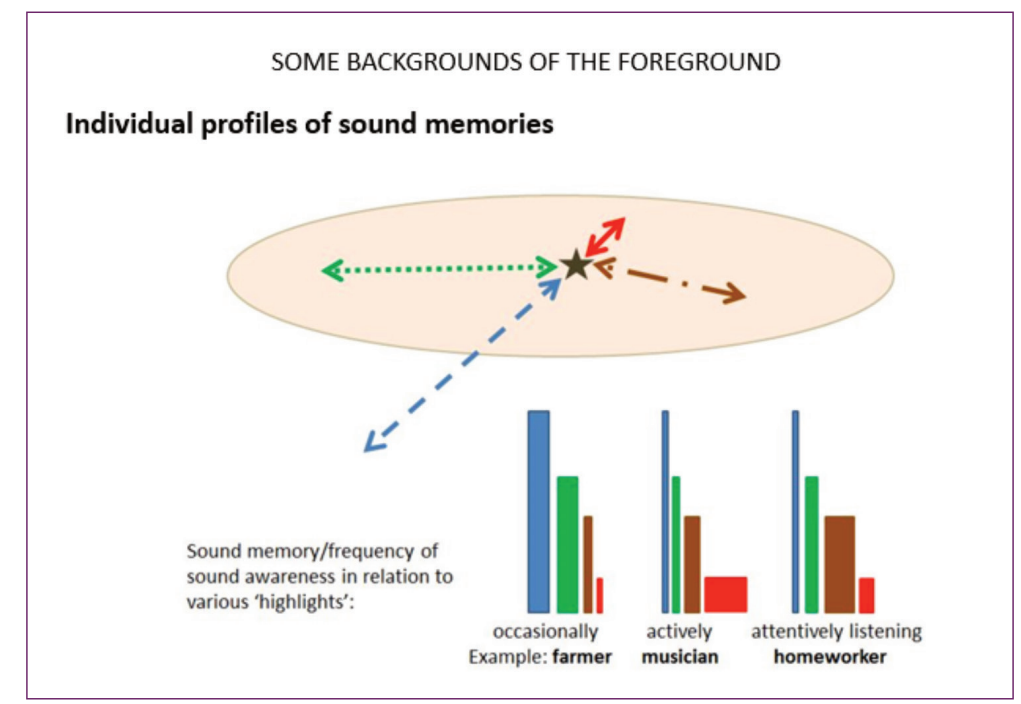

Figure II: Individual profiles of sound memories.

The fore-/background aspect can be taken as a point of departure to explain this independently existing inter-connectivity between listening habits, main 'profession', and individual spectrum of activities

To give a parallel example:Visual depictions always include background and details of objects that are not in the main focus. Only the visual context makes a drawing or painting informative and enjoyable. Sketches of single details are considered incomplete. What about sound?

Or another example:The dancer is foreground, the musicians are background. That may apply on the visual aspect. What about the sound? Also, the dancer's sound is not arbitrary. There are bangles, steps on the bamboo flooring, and there are expressions of appreciation on the side of those who watch the performance. The idea of an 'ecological framework' becomes obvious, if any of these sounds is cut off.

One of the most striking background-sounds is the sound of walkers on the longhouse flooring. To the people of Annah Rais, this sound is as natural as the wind, the water, the birds and frogs around. Recently, I could observe a very interesting documentary made by Juan Javier Rivera on a recording situation in South America. People were gathered in the darkness of the night and all possibly 'disturbing' sound was tediously avoided. The documentary finishes with not having recorded anything. Looking at these two cases, the absurdity of 'cleaning up' becomes obvious. The outcome is not 'clean', it is incomplete.

5 Pratuokng is a tube zither with 5- I I strings made of skin-stripes of the same bamboo tube (Jähnichen \& Musib 2013). 
On the map below, the performance area and the usual areas of daily activities are drafted. They mark not only different distances but different experiences of sound.

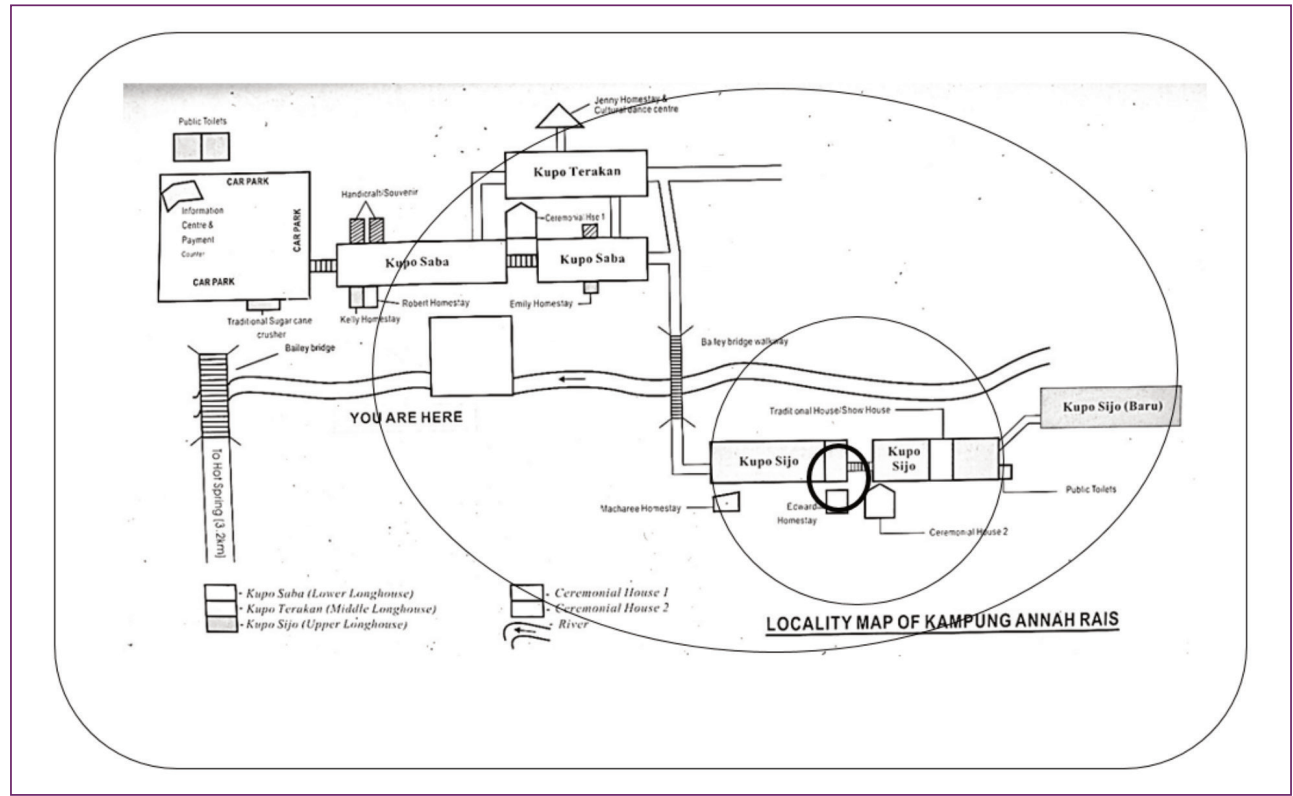

Figure 12: Map with auditory zones.

In the experiment, the author and his friends were simulating space categories that are captured simultaneously to provide a choice of perspectives. That delivers an uncompromised picture of sound experiences.

Following the suggestions implied in this experiment, we could be able to hear, how a place sounded 20 years ago at lunch time or at night, we could hear how it sounds if moving in different directions as if having 3D visuals with real surround sound. What if additionally to the visual invitation to live at a place, to visit a concert, to walk through a shopping mall or through a park, the sound could be provided as it is perceived from various 'highlights'? Technically, these options are possible and attract some attention. It is the understanding of a need for the ecology of sound in life that is certainly missing. In consequence, the understanding of a need for audiovisual archives with qualified staff is partly missing as well.

Sound indicates time and space. Places live with sound. Sound is one important attribute of time and space. It can be a tool for time and space recognition. Music is a distinct sound within this sounding world. Also, the experiment indicates that villages are not quiet. Cities are not noisy. Quietness and noise are relative. It is the meaning one gives them that makes them quiet or noisy. These and many other aspects can play a role in finding auditory shapes, auditory meanings, and auditory changes.

Essentially, it is time to put any type of distinct sound, including all that what is called music, into a wider context of perception. Audiovisual archivists can pioneer in applicative sound ecology. 
Summarizing the findings and observations so far:

- The way how recordings of pratuokng and other local instruments are made does not fit any of the given 'contextual sound profiles'.

- While playing an instrument is not confined to the instrument's body, the sound source is mutually changing its actual context.

- The contextual sound defines to a high percentage a spatial arrangement of sound sources with and without focus.

- In order to preserve sound, its sound context has to be preserved as well through spatial, temporal, and individual options.

- Temporality becomes increasingly a factor for contextual sound preservation as time influences all sound in which the main sound source is embedded.

- Memory draws on spatial hearing as well as on recognizing moving sound in a perceptible time period.

- Sound isolation as experienced in high tech performance venues deletes temporal and spatial features.

- This is caused through 'pretending spaceless eternity'.

- The only leftovers from an authentic experience is the audience trying to keep quiet.

- However, most of the memorable context to any musical sound in focus is context indicating and includes temporal and spatial features.

- Today, preserving music could technically and should ethically include its sound context to keep not only individual memories alive. Any preservation efforts will have to include the aspect of 'sound life' around its main target.

- Only then it is possible to explore a 'musical life' apart from time and space.

Another way to attract attention is an interdisciplinary embedding of skills and knowledge into a wider academic and industrial context and to connect them with the communities affected. In result, this approach helps a lot in promoting not only sound studies but awareness about historical sound events and future possibilities of preserving contemporary sound.

\section{Some thoughts and an experiment}

In the context of earlier sound production, i.e., we may rise the question "How did people hear the real thing?" and try to project a possibility of how one can listen back into the past. An experiment with parallel digital and analogue recordings may try to figure out how the real music behind the recordings on now obsolete carriers might have been. The idea derives from a few simple thoughts: If one can produce a profile of unwanted sound and subtract it in sum from a recording, there should be also the opposite way: One should be able to detect a 'wanted' sound and add in sum to a recording. Today, there might be a possibility to detect in synchronous live recordings the principal differences between widely obsolete and high standard digital recordings. The findings can lead to an applicable algorithm or a model tool that helps to recreate a near to true sound environment which could be heard live in the past when these obsolete carriers were still standard carriers. This aspect plays an important role in a wider concept of preservation applied on postcolonial cultures with remarkable discontinuities in social developments.

The fast market turnover of recording formats in the past ten years makes current material often obsolete (Seeger, 2007: 00:00:00-00:00:30). Carriers used for recordings in the twentieth century might not be played back in the twenty-first century due to systems and format incompatibility of playback and recording devices. On the other hand, current technology can restore sound from whatever carrier more and more reliably. Overall, audiovisual preservation can be generalized into two main necessities. The needs are preservation of the medium that holds the content, and the preservation of the content itself. Most of the medium such as wax cylinder, vinyl's or phonograph, open-reels, cassette tapes, mini discs, compact discs, tapes in the video home system orVHS and all other audiovisual carriers are unstable (Musib, 20 1 5:33-34). Therefore the need for migration of audio and visual materials to more stable media is essential."The process of signal acquisition in real-time, may lead to some constraint and that is 
time. Ethical issues and quality should never be bargained" (Wallaszkovitz, 2012:ARCPA 2053, perf). Monitoring every second of sound may be tedious, but neglecting the process may lead to poor signal extraction (Musib, 2015:33-34).

In addition to physical preservation, conservation efforts towards preserving the content of audio and visual recordings of historical events of the past and future have always a priority. To name a few research articles regarding future thoughts of audio preservations from the perspectives of context were Musib in his thesis regarding contextual sound preservation through collecting sound via 'technical highlights', Umashankar Mantravadi's article "Survival of traditional performance under different acoustic conditions" presented at the 43rd International Association of Sound and Audiovisual Archives (IASA) Conference in New Delhi, India, in which he reported on documenting and archiving acoustic properties of an old temple used as a theatre with the aid of 'impulse response' as a tool. Other articles appeared in the context of city design and architecture (Jones 2005).

\section{Methods used in the experiment}

The research applies qualitative research methods, especially recording experiments leading to a descriptive analysis through testing audio recordings. In the process, simultaneous audio recordings were made on different carriers such as the normal and chrome bias cassettes tapes, and a digital audio recording of $44 \mathrm{kHz}$ sampling rate (minimum) utilizing appropriate audio recording equipment that is also partially examined in this study. The audio analysis focuses descriptively on the aspect of differences in audio quality. The sound captured represents two different perspectives, namely the sound of the musical instruments recorded using cassette tapes, and the digital recording which represents the entire sound setting in a place as it can be heard using the currently most advanced technology.

The test was conducted in two different environments of which one is a practice room, and another is a lecture hall. The purpose of using these two different environments is to have a constant ambience and its noise floor as the backdrop of the overall sound program for both recording experiments. This constant ambience and its noise floor are captured and defined as sound profiles. Two sound profiles were collected via two different formats.

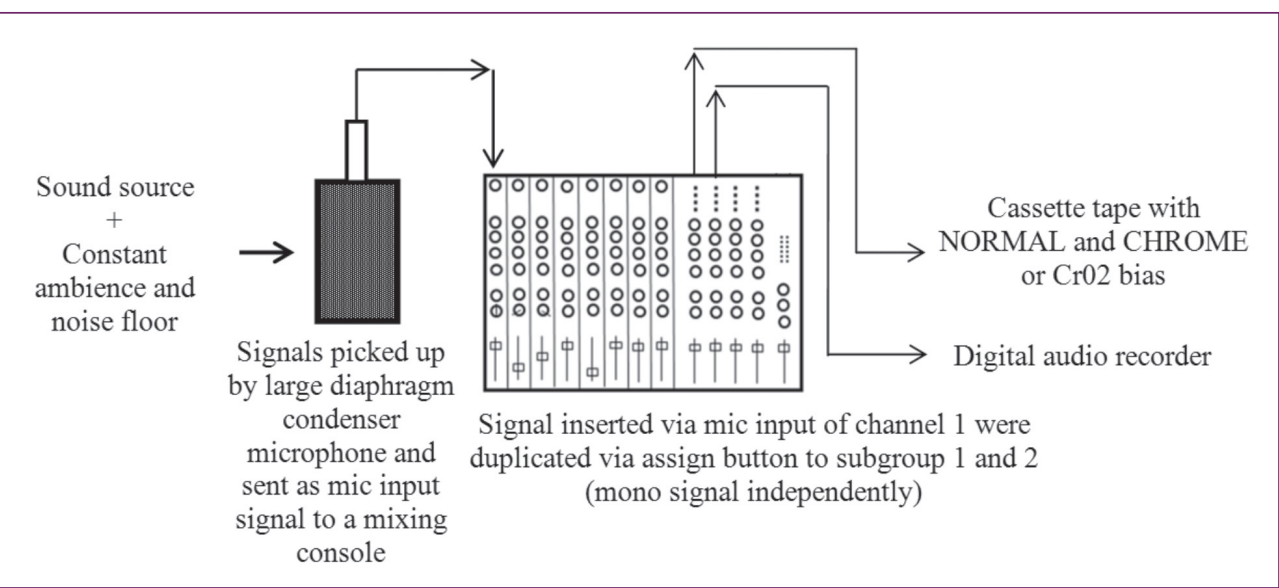

Figure I3:An experiment conducted through parallel recording of a 'bansuri' on two different formats namely the cassette tapes and the digital audio recorder.

The experiment conducted based on the set-up above is purely a simulation to recreate what is actually being heard and then controlled through the representation of the digital audio recording compared to what was collected during field work from the cassette tapes in the past. The signal had to be captured by a large diaphragm condenser microphone and recorded 
separately on two recording devices. This was done through a mixer with all equalizations and effects, send and return, set to by-pass. The outcomes of both recordings were analyzed through amplitude analysis tools.

\begin{tabular}{|l|l|l|l|}
\hline Descriptions & $\begin{array}{l}\text { NORMAL } \\
\text { BIAS }\end{array}$ & $\begin{array}{l}\text { CHROME } \\
\text { BAIS }\end{array}$ & $\begin{array}{l}\text { DIGITAL } \\
\text { AUDIO 24 BIT }\end{array}$ \\
\hline Peak Amplitude & -12.11 & -13.69 & -20.57 \\
\hline Maximum Sample Value & 2079675 & 1734188 & 784298 \\
\hline Minimum Sample Value & -2051573 & -1636108 & -785377 \\
\hline Possibly Clipped Samples & 0 & 0 & 0 \\
\hline Total RMS Amplitude & -21.39 & -25.28 & -30.45 \\
\hline Maximum RMS Amplitude & -16.39 & -19.74 & -24.32 \\
\hline Minimum RMS Amplitude & -60.07 & -61.19 & -71.26 \\
\hline Average RMS Amplitude & -24.28 & -28.46 & -33.52 \\
\hline DC Offset & $0.00 \%$ & $0.00 \%$ & $0.00 \%$ \\
\hline Measured Bit Depth & 24 & 24 & 24 \\
\hline Dynamic Range & 43.68 & 41.45 & 46.94 \\
\hline Dynamic Range Used & 42.45 & 38.90 & 41.20 \\
\hline Perceived Loudness & -17.69 & -21.44 & -25.41 \\
\hline Perceived Eq Loudness & -14.31 & -16.65 & -22.39 \\
\hline
\end{tabular}

Figure 14:Amplitude analysis of the bansuri from the perspective of normal, chrome and DAW (digital audio workstation) recording.

The table shows the general amplitude analysis of the parallel recordings. The differences are audible through these tapes as their bias characteristics were different. The table indicates differences in total RMS amplitude across all formats, showing the total power of the entire selection with normal bias being a higher value at $-21.39 \mathrm{~dB}$ compared to chrome at $-2528 \mathrm{~dB}$ and digital format -30.45 which seems to be much softer in its level of loudness. Similar to the perceived loudness or the average RMS power,all formats seem to decrease from - 17.69dB for normal bias, $-2 \mathrm{I} .44 \mathrm{~dB}$ for chrome and $-25.4 \mathrm{IdB}$ for digital audio. There are many factors which contribute to this reading. Tape hiss and noise floor were the two main contributors to the overall loudness containing in the constant ambience.

These audible differences are obvious particularly while working in a recording studio. Signals that were send out to a recording device are very much what one would get back as 'tape return' signals that seem to be the same. Analogue recordings can be, therefore, attractive to a listener from certain aspects that reflect their own sound ideals. To an audio engineer, it might be pleasing to have the assurance of knowing that any signal one put in as 'tape send signal' into a DAW is closed to what you get out of it.

The following is the graphic representation of constant ambience and its noise floor as the backdrop of the bansuri played and recorded on a normal bias tape.Visualization in logarithmic pane enables one to view the frequency spectrum of the constant ambience within the range of 20 $\mathrm{Hz}$ to $20 \mathrm{kHz}$. The frequencies ranging from $200 \mathrm{~Hz}$ to $20 \mathrm{kHz}$ were concealed by the threshold. 


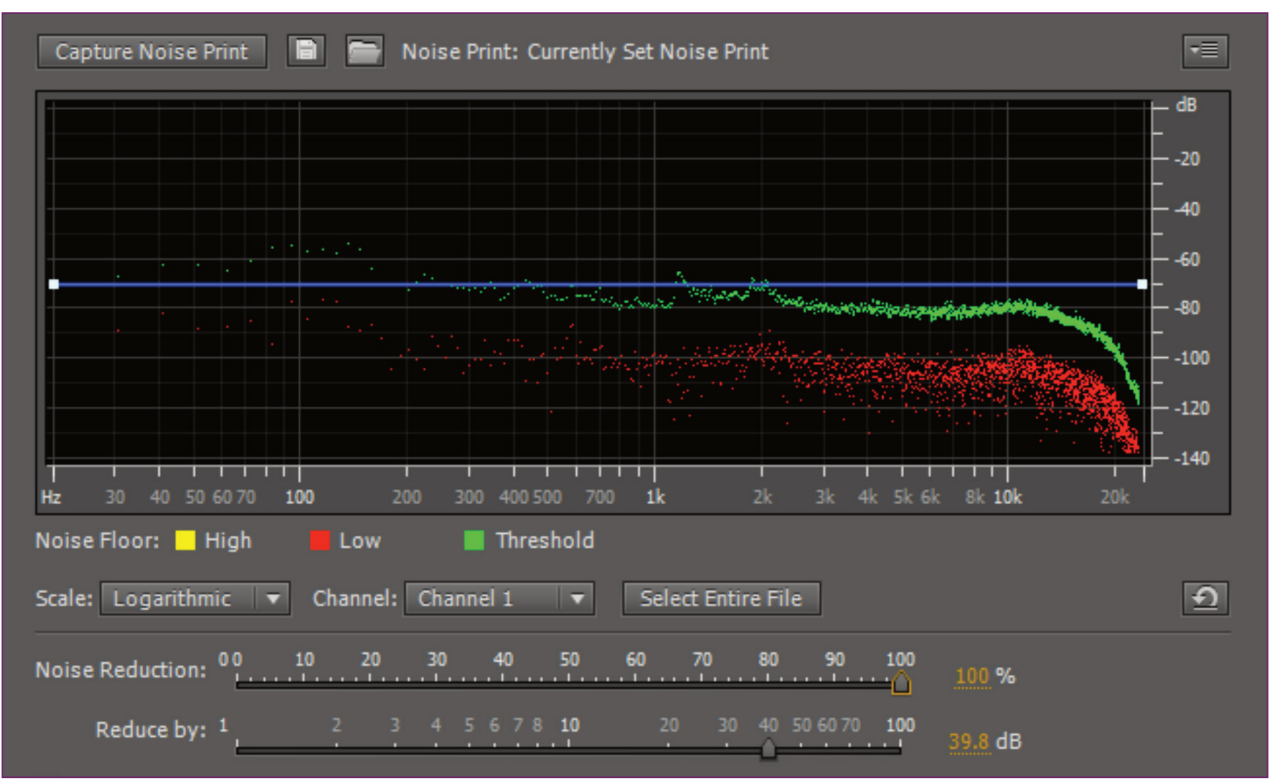

Figure 15:The frequencies ranging from $200 \mathrm{~Hz}$ to $20 \mathrm{kHz}$ were concealed by the threshold.

Figure 3 is the graphic representation of constant ambience and its noise floor as the backdrop of the bansuri played and recorded on a normal bias tape with the threshold value set to $50 \%$.

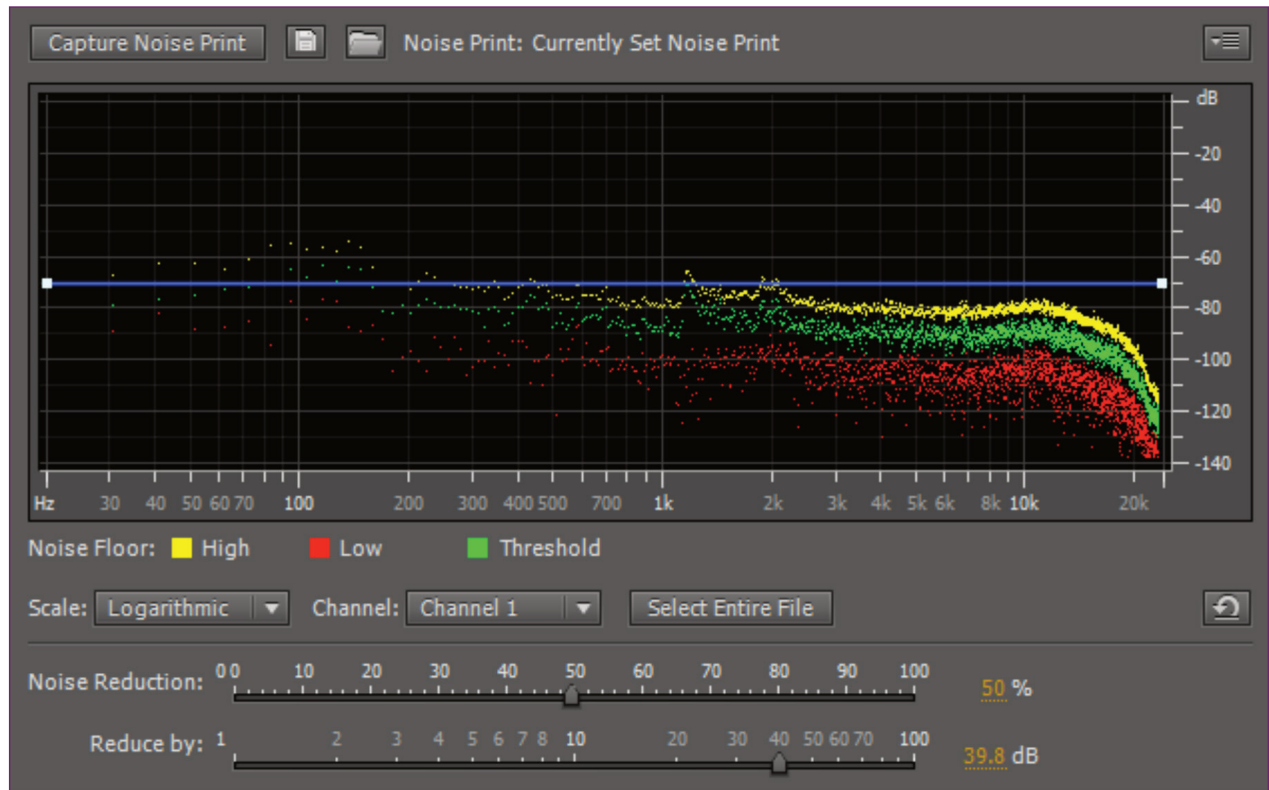

Figure 16:The frequencies ranging from $200 \mathrm{~Hz}$ to $20 \mathrm{kHz}$ with threshold reduce by $50 \%$. 
The captured constant ambience as 'noise print' used against the bansuri recorded on a normal tape bias yield a bansuri with constant ambience removed. The process is not only removing the ambience before the waveform starts, but within the bansuri harmonic spectrum too.
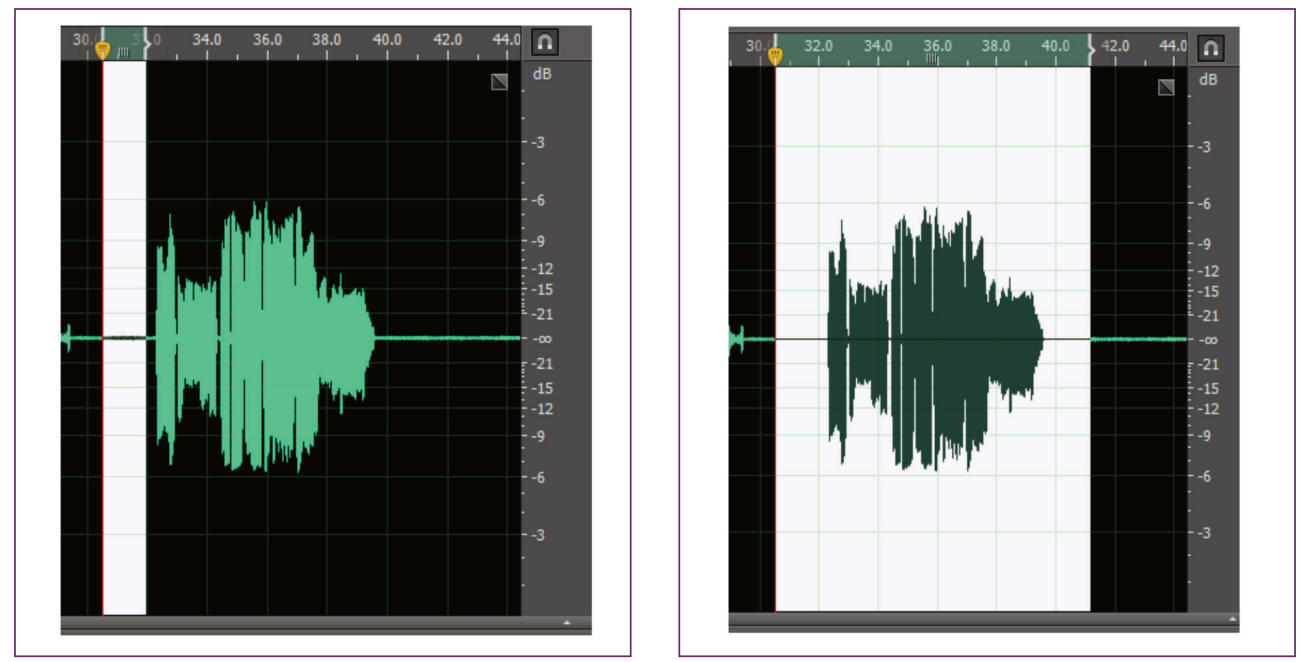

Figure 17: Bansuri waveform on the left illustrate the capturing of the constant ambience found as a backdrop in the normal bias tape. On the right is the bansuri of the same tape with the constant ambience removed. Notice the green center line on the highlighted region is thinner compare to the one on the left.

The constant ambience remnants comprises of mostly tape hiss and the upper harmonics of the bansuri and the ambience. The experiment has its limitation when it comes to uncontrolled dynamic range that involves the representation of the softest signal where background noise becomes a distraction and to the loudest signal where signals begins to overmodulate and to be distorted. Some of the field recording collected by researchers during the tape era contain technical defects such as electrical buzzing, humming, low rumble noise due to faulty connections of microphones; or the recording signal was set too high that causes over-modulation. Although the process to remove these defects can be conducted, it is time consuming and the outcome would just be of a similar bad quality pretending less technical defects.

With regards to other carriers such as the vinyls, signal extraction to another medium such as digitization may incur issues. Particularly materials in which the content is of a concept album, for example, The Beatles in their Sergeant Pepper Lonely Hearts Club Band album entitled A Day in the Life ${ }^{6}$ composed by John Lennon and Paul McCartney. George Martin wrote in the program notes of the album synopsis that in the piece after the last crashing piano chords, he had a few seconds of $15 \mathrm{kHz}$ tone generated just to annoy your dogs, few seconds of Beatle chatter, taped, cut into several pieces and paste them back together in random order. He says "This was done on purpose so that to those who bought the vinyl and play it on a non-auto return player, would create a continuous noise until it run out of groove effect" (quoted after Musib 20I2, I58).

In this case signal extraction would have to be done from the original master tape. Signal extraction out of the vinyl itself may face other issues such as constant static, pops and crackling aside from restoring other issues such as noise and electrical hums. On some occasions, this would harm the vinyls. 


\section{Final remarks}

All these research based findings are to attract interest in the very essential first business: to establish, maintain and develop audiovisual archives that care about their substantial existence for the community, the entire sound ecology within a place, and its primary quality.

Philosophically, it is not about modifying the original recording but about recreating the sound information provided through it in a new data object. Thinking further in this line, this process might be employed again in a couple of decades on recordings produced today.

This or similar ways to approach research partners and promoting AV archiving is an elementary condition in not only serving the community but possibly in giving opportunities for applications beyond contemporary academia.

It is first about promoting the features of sound restoration, sound re-positioning, and sound imaging rather than promoting the philosophy of preservation behind, and only in second instance it is a holistic picture of different preservation issues. Some essential details of the planned teaching course and the way of attracting an increasing interest in AV archiving and preservation issues - exemplarily under Malaysian circumstances - should invite discussion among those dedicated to training and education in IASA.

\section{References}

Kwon, Heonik (2012). Perspectivism in Social Anthropology. CultureNature I, 59-68.

Jähnichen, Gisa \& Ahmad Faudzi Musib (20I3). Social Scientists as Users: Searching for Recorded Sound in its Environment. IASA Journal, 40, pp. 44-54.

Jones, Patrick M. (2005). Music Education and the Knowledge Economy: Developing Creativity, Strengthening Communities, Arts Education. Policy Review, 106 (4), pp. 5-I2.

Manthravadi, Umashankar (20I2). Archiving Acoustics. Paper presented at the 43rd IASA Annual Conference 2012, New Delhi, India, 7-II October 2012. Manuscript.

Musib, Ahmad Faudzi (20I I). Sound Print as an Identification Tool. Music and Memory. Edited by Gisa Jähnichen \& Julia Chieng. UPM Book Series on Music Research, 4. Serdang: UPM Press, ISSN 2289-3938, pp. I57-I 70.

Musib, Ahmad Faudzi, Gisa Jähnichen and Chinthaka Prageeth Meddegoda (20I4). The Audiovisual Research Collection for Performing Arts (ARCPA) at Universiti Putra Malaysia: Negotiating Ethical Issues in Social Sciences. IASA Journal, 43, pp. 53-59.

Seeger, Anthony (2007). Ethnomusicologist Anthony Seeger on Preserving Audio Recordings [documentary]. ArtisthouseMusic. Accessible via URL: http://www. artistshousemusic.org/videos/ethnomusicologist+anthony+ seeger+on+preserving+ audio+recordings (Retrieved 5 January 2013).

Wallaszkowitsz, Nadja (20I2). Providing Access to Research Sound Collections: Quick and Dirty or Highest Quality. Paper presented at the 43rd IASA Annual Conference 20I2, New Delhi, India, 7-I I October 2012. Manuscript.

\section{Audio}

“Day in the Life". The Beatles (1967). Sgt. Pepper's Lonely Hearts Club Band. E.M.I. Records CDP 7464422. 